\title{
Effects of trap anisotropy on impurity scattering regime in a Fermi gas
}

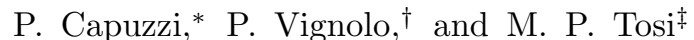 \\ NEST-INFM and Classe di Scienze, Scuola Normale Superiore, I-56126 Pisa, Italy
}

\begin{abstract}
We evaluate the low-lying oscillation modes and the ballistic expansion properties of a harmonically trapped gas of fermionic ${ }^{40} \mathrm{~K}$ atoms containing thermal ${ }^{87} \mathrm{Rb}$ impurities as functions of the anisotropy of the trap. Numerical results are obtained by solving the Vlasov-Landau equations for the one-body phase-space distribution functions and are used to test simple scaling Ansatzes. Starting from the gas in a weak impurity-scattering regime inside a spherical trap, the time scales associated to motions in the axial and azimuthal directions enter into competition as the trap is deformed to an elongated cigar-like shape. This competition gives rise to coexistence of collisionless and hydrodynamic behaviors in the low-lying surface modes of the gas as well as to a dependence of the aspect ratio of the expanding cloud on the collision time.
\end{abstract}

PACS numbers: 03.75.Ss, 02.70.Ns

\section{INTRODUCTION}

Experiments on ballistic expansion and collectivemode excitations have provided important diagnostic tools in the study of quantum-degenerate atomic and molecular gases (for a recent review see [1]). A milestone example is the interpretation of the anisotropic expansion of a cloud of ultracold bosons as the smoking gun of Bose-Einstein condensation in a trapped dilute gas 2, 3, 4]. The later achievement of degenerate Fermi gases [5] and boson-fermion mixtures [6, 7, 8] has opened up the possibility to experimentally observe rich quantum phase diagrams and to study the interplay between external confinement and interspecies collisions. More recently, the expansion behavior of strongly interacting fermion clouds has been the object of experiments aimed at probing novel superfluid states on the crossover from a BCS superfluid to a condensate of molecular dimers 9, 10, 11].

Collective modes provide a direct measure of the collisionality of a dilute quantum gas. Monopolar and quadrupolar modes have been proposed as markers of the approach of quantum phase transitions in binary mixtures of Bose-Einstein condensed gases (BEC's) 12], in boson-fermion mixtures [13, 14], and in fermion mixtures across the BCS-BEC crossover [15, 16, 17]. The dynamical transition from collisionless to hydrodynamic behavior in fermion mixtures has been followed experimentally 18] and numerically [19] in two-component fermion mixtures by studying their dipolar oscillation modes. The role played in this context by mobile impurities inside a fermion gas under spherical confinement has also been studied by numerical means [20].

In the present work we examine how the anisotropy of the trap affects the low-lying oscillation modes and the ballistic expansion of a gas of fermionic ${ }^{40} \mathrm{~K}$ atoms

\footnotetext{
*Electronic address: capuzzi@sns.it

${ }^{\dagger}$ Electronic address: vignolo@sns.it

${ }^{\ddagger}$ Electronic address: tosim@sns.it
}

containing a small concentration of thermal ${ }^{87} \mathrm{Rb}$ atoms. We numerically solve the Vlasov-Landau equations for the evolution of the phase-space distribution functions within a particle-in-cell approach and compare the results with simple scaling Ansatzes. Our results demonstrate that in a cigar-shaped harmonic confinement, where two different confinement frequencies determine two different time scales, collisionless and hydrodynamic behaviors can coexist in the low-lying collective excitations of the gas and that the aspect ratio of the expanding cloud shows a non-monotonic dependence on the anisotropy of the trap.

The paper is organized as follows. In Sect. III we introduce the mixture under study and the basic equations that will be used to describe its dynamics. Section III analyzes the monopolar, dipolar, and quadrupolar oscillations of the gas as functions of the anisotropy of the confining potential, while Sect. IV gives a discussion of the dynamics of the free expansion of the fermion cloud. Finally, Sect. $\nabla$ presents a summary and the main conclusions of our work.

\section{THE MIXTURE}

We consider a gas of fermionic atoms of mass $m_{F}$ confined inside an axially symmetric harmonic trap of the form

$$
V_{F}(\mathbf{r})=\frac{1}{2} m_{F} \omega_{F, \perp}^{2}\left(x^{2}+y^{2}+\lambda^{2} z^{2}\right)
$$

where $\omega_{F, \perp}$ is the angular trap frequency for motions along the $\hat{x}$ and $\hat{y}$ directions and $\lambda=\omega_{F, z} / \omega_{F, \perp}$ is the anisotropy parameter. Potentials with $\lambda \ll 1$ generate cigar-shaped density profiles. The fermions are wholly spin-polarized and hence at very low temperature the Pauli principle quenches collisions among them. A second component must then be added in order to thermalize the gas and to drive its collisionality.

We dope the fermion gas with a small number of bosons having larger atomic mass $m_{B}$. We simply think of them 
as impurities confined by the harmonic potential

$$
V_{B}(\mathbf{r})=\frac{1}{2} m_{B} \omega_{B, \perp}^{2}\left(x^{2}+y^{2}+\lambda^{2} z^{2}\right),
$$

where $\omega_{B, \perp}$ is the angular trap frequency in the azimuthal plane and we have assumed that bosons and fermions share the same trap anisotropy $\lambda$. The difference in atomic masses and particle numbers causes a much lower quantum degeneracy temperature for the bosons and we can therefore consider them as an uncondensed cloud, even in the presence of highly degenerate fermions.

The dynamics of the mixture is described through the one-body distribution functions $f^{(F, B)}(\mathbf{r}, \mathbf{p}, t)$ in the Boltzmann approximation. Their evolution is governed by the Vlasov-Landau kinetic equations (VLE),

$$
\partial_{t} f^{(j)}+\frac{\mathbf{p}}{m_{j}} \cdot \nabla_{\mathbf{r}} f^{(j)}-\nabla_{\mathbf{r}} U^{(j)} \cdot \nabla_{\mathbf{p}} f^{(j)}=C\left[f^{(F)}, f^{(B)}\right]
$$

where the Hartree-Fock effective potential is $U^{(j)}(\mathbf{r}, t)=$ $V_{j}(\mathbf{r})+g n^{(\bar{j})}(\mathbf{r}, t)$ with $\bar{j}$ denoting the species different from $j$. Here we have set $g=2 \pi \hbar^{2} a / m_{r}$ with $a$ being the $s$-wave scattering length of a fermion-boson pair and $m_{r}$ its reduced mass, and $n^{(j)}(\mathbf{r}, t)$ is the spatial density given by integration of $f^{(j)}(\mathbf{r}, \mathbf{p}, t)$ over the momentum degrees of freedom. Since we deal with low concentrations of impurities we have neglected impurity-impurity interactions. In addition, collisions between spin-polarized fermions are negligible at low temperature and thus the collision integral $C$ in Eq. (3) involves only collisions between fermions and impurities. This is given by

$$
\begin{aligned}
C= & \frac{\sigma}{4 \pi(2 \pi \hbar)^{3}} \int d^{3} p_{2} d \Omega_{f} v\left[\left(1-f^{(F)}\right)\left(1+f_{2}^{(B)}\right) f_{3}^{(F)} f_{4}^{(B)}\right. \\
& \left.-f^{(F)} f_{2}^{(B)}\left(1-f_{3}^{(F)}\right)\left(1+f_{4}^{(B)}\right)\right],
\end{aligned}
$$

where $f^{(j)} \equiv f^{(j)}(\mathbf{r}, \mathbf{p}, t)$ and $f_{i}^{(j)} \equiv f^{(j)}\left(\mathbf{r}, \mathbf{p}_{i}, t\right), d \Omega_{f}$ is the element of solid angle for the outgoing relative momentum $\mathbf{p}_{3}-\mathbf{p}_{4}, v=\left|\mathbf{v}-\mathbf{v}_{2}\right|$ is the relative velocity of the incoming particles, and $\sigma=4 \pi a^{2}$ is the scattering cross-section. The collision satisfies conservation of momentum $\left(\mathbf{p}+\mathbf{p}_{2}=\mathbf{p}_{3}+\mathbf{p}_{4}\right)$ and energy $\left(\varepsilon+\varepsilon_{2}=\varepsilon_{3}+\varepsilon_{4}\right)$, with $\varepsilon_{j}=p_{j}^{2} / 2 m_{j}+U^{(j)}$.

The solution of Eqs. (3) is carried out by using a numerical approach based on particle-in-cell plus Monte Carlo sampling techniques, which allows us to evaluate the kinetics of such systems down to $T \simeq 0.1 T_{F}$. The technical details of the method have been given elsewhere [19, 20, 21].

In the following we shall focus on the dependence of the dynamics of a mixture of $N_{F}=10^{4}{ }^{40} \mathrm{~K}$ atoms and $N_{B}=10^{2}{ }^{87} \mathrm{Rb}$ atoms on the anisotropy $\lambda$ of the traps at fixed values of the average trap frequencies $\bar{\omega}_{F}=\left(\omega_{F, \perp}^{2} \omega_{F, z}\right)^{1 / 3}$ and $\bar{\omega}_{B}=\left(\omega_{B, \perp}^{2} \omega_{B, z}\right)^{1 / 3}$. These are taken as the geometric averages of the trap frequencies in the experiments carried out at LENS on ${ }^{40} \mathrm{~K}-{ }^{87} \mathrm{Rb}$ mixtures [22], i.e. we set $\bar{\omega}_{F}=2 \pi \times 134.1$ $\mathrm{s}^{-1}$ and $\bar{\omega}_{B}=2 \pi \times 91.2 \mathrm{~s}^{-1}$. We fix the temperature at $T=0.2 T_{F}$, with $T_{F}=\hbar \bar{\omega}_{F}\left(6 N_{F}\right)^{1 / 3} / k_{B}$ being the Fermi degeneracy temperature for noninteracting fermions. This also corresponds to $T \simeq 2.7 T_{\mathrm{BEC}}$ where $T_{\mathrm{BEC}}=0.94 \hbar \bar{\omega}_{B}\left(N_{B}\right)^{1 / 3} / k_{B}$ is the condensation temperature for the noninteracting Bose component. Finally, we assume a repulsive $s$-wave scattering length $a=2000$ Bohr radii. This choice of the fermion-boson scattering length is dictated by computational convenience, but still leaves the mixture at $\lambda=1$ in a collisionless-tointermediate scattering regime as for the ${ }^{40} \mathrm{~K}-{ }^{87} \mathrm{Rb}$ mixtures studied at LENS.

\section{OSCILLATION MODES}

\section{A. Dipolar oscillations}

The collisional state of the gas is revealed by the behavior of the frequencies and damping rates of collective excitations as functions of the collision frequency. Briefly, for dipolar modes the hydrodynamic behavior is signalled by a common frequency of oscillation of the two species and by a decrease of the damping rate with increasing collision frequency. Conversely the collisionless regime, which is attained at low collisionality, is characterized by different oscillation frequencies and by growing damping rates. The collision rate can be evaluated either from a numerical simulation which actually counts the number of collisions at each time step, or by direct integration of the collision integral over momenta. Pauli blocking strongly quenches collisions at the temperatures of present interest and its handling in numerical studies requires suitably adapted methods of Monte Carlo sampling [19, 20, 21].

In the numerical simulation we excite dipolar modes by initially shifting the fermionic density profile by $a_{h o}=$ $\sqrt{\hbar /\left(m_{F} \omega_{F, \perp}\right)}$ in either the axial or radial direction ( $\hat{z}$ and $\hat{x}$, say) and then fit the time evolution of the fermionic center-of-mass coordinates with the functions $\cos \left(\Omega_{i} t+\phi\right) \exp \left(-\gamma_{i} t\right)$. From these fits we extract the oscillation frequencies $\Omega_{i}$ and the damping rates $\gamma_{i}$ along the two directions. The results obtained from the numerical solution of the Vlasov-Landau equations are shown in Fig. 11 The error bars in the plot have been estimated from the standard deviations of $\Omega_{i}$ and $\gamma_{i}$ in the fitting process.

Starting from $\lambda=1$ and increasing the anisotropy towards an elongated cigar-shaped trap, the frequencies of the dipolar oscillations show different behaviors along the axial and the radial direction. The value of $\Omega_{\perp} / \omega_{F, \perp}$ increases slightly towards unity as $\lambda$ is decreased, whereas $\Omega_{z} / \omega_{F, z}$ decreases with $\lambda$ towards an appreciably lower value. At the same time both damping rates tend to vanish, although that for axial oscillations appears to go through a broad maximum before doing so. These behaviors indicate that in strongly elongated traps the dynamics of the Fermi gas is collisionless in radial dipolar 
oscillations, but collisional in axial ones.

The coexistence of collisionless and hydrodynamic behaviors in the dipolar oscillations as presented above is supported by the solution of scaling equations in the classical limit. Within a classical model one can write a set of equations for the coupled motions of the centers of mass of fermions and bosons in the small-oscillation regime 18, 22. If we include mean-field effects, the equations of motion in the $i$-direction for the center-of-mass coordinates and for the relative coordinates are

$$
\left\{\begin{aligned}
\ddot{x}_{\mathrm{CM}, i} & =-\omega_{\mathrm{hd}, i}^{2} x_{\mathrm{CM}, i}-M_{r} \Delta \omega^{2} x_{r, i} / M \\
\ddot{x}_{r, i} & =-\Delta \omega^{2} x_{\mathrm{CM}, i}-\omega_{r, i}^{2} x_{r, i}+\omega_{\mathrm{mf}, i}^{2} x_{r, i}-\omega_{Q} \dot{x}_{r, i}
\end{aligned}\right.
$$

where $x_{\mathrm{CM}, i}=\left(m_{F} N_{F} x_{F, i}+m_{B} N_{B} x_{B, i}\right) / M$ and $x_{r, i}=$ $x_{F, i}-x_{B, i}$. We have defined $\Delta \omega^{2}=\omega_{F, i}^{2}-\omega_{B, i}^{2}$ and $\omega_{r, i}^{2}=\left(m_{B} N_{B} \omega_{F, i}^{2}+m_{F} N_{F} \omega_{B, i}^{2}\right) / M$ with $M=m_{F} N_{F}+$ $m_{B} N_{B}$ and $M_{r}=m_{B} m_{F} N_{B} N_{F} / M$. The hydrodynamic frequencies are given by

$$
\omega_{\mathrm{hd}, i}=\left(\frac{m_{F} N_{F} \omega_{F, i}^{2}+m_{B} N_{B} \omega_{B, i}^{2}}{M}\right)^{1 / 2}
$$

and the collisional frequency is $\omega_{Q}=4 Q\left(m_{B} / N_{F}+\right.$ $\left.m_{F} / N_{B}\right) /\left(3 m_{F}+3 m_{B}\right), Q$ being the total number of collisions per unit time defined as

$$
Q=\frac{\sigma}{\pi^{2}} \frac{N_{F} N_{B}}{k_{B} T}\left(\frac{k_{x} k_{y} k_{z}}{m_{r}}\right)^{1 / 2} .
$$

In Eq. (7) $k_{i}=k_{F, i} k_{B, i} /\left(k_{F, i}+k_{B, i}\right)$ are the effective oscillator constants, with $k_{j, i}=m_{j} \omega_{j, i}^{2}$. In the large- $Q$ limit Eqs. (5) predict that the relative motion of the two clouds is overdamped, so that fermions and bosons oscillate together at the hydrodynamic frequencies. For the system parameters that we are using these are $\omega_{\mathrm{hd}, i}=0.9942 \omega_{F, i}$. While due to the low number of impurities the value of $\omega_{\mathrm{hd}, i}$ is very close to the bare trap frequency $\omega_{F, i}$, their difference can be amplified by, e.g., increasing the mass of the impurities or varying the trapping frequency.

The mean-field correction to the bare frequency of the relative motion in Eq. (5) is

$$
\omega_{\mathrm{mf}, i}^{2}=\frac{g}{M_{r}} \int d^{3} r \frac{\partial n_{0}^{(F)}}{\partial x_{i}} \frac{\partial n_{0}^{(B)}}{\partial x_{i}},
$$

where $n_{0}^{(F, B)}(\mathbf{r})$ are the equilibrium density profiles. Equation (8) extends the result of Ref. 23] to a general gaseous mixture. According to Eqs. (5) the mean-field correction is more important in the collisionless limit and does not affect the value of the hydrodynamic frequency. However, it shifts the value of $Q^{\text {lock }}$ at which the two clouds become glued together. The locking point can be estimated by looking for an overdamped oscillation in the solution for the relative motion in Eqs. (5). If we neglect the coupling between the center-of-mass and the relative motions we find

$$
\left.\sqrt{\omega_{r, i}^{2}-\omega_{\mathrm{mf}, i}^{2}} \simeq \frac{\omega_{Q}}{2}\right|_{Q^{\text {lock }}} .
$$

We have estimated a value of $\omega_{\mathrm{mf}, i}^{2} / \omega_{F, i}^{2} \sim 8 \times 10^{-3}$ for the isotropic trap and verified that this ratio is slightly decreasing with $\lambda$. Therefore the mean-field correction is negligible in the present case and the locking point is fixed by the bare frequency of the relative coordinate, namely $\left.\omega_{Q}\right|_{Q^{\text {lock }}} \simeq 2 \omega_{r, i} \simeq 1.4 \omega_{F, i}$.

The classical model does not contain the effects of Pauli blocking at low temperature, so that we have taken $Q$ as a single fitting parameter at all values of $\lambda$. The results obtained from Eqs. (5) with $Q=55 \bar{\omega}_{F}$ are reported in Fig. [1 and compared with the numerical results for the dependence of the mode frequencies and damping rates on the anisotropy parameter. At $\lambda=0.05$ the gas is already very close to the hydrodynamic regime in its motions along $\hat{z}$, since the value of the oscillation frequency obtained in the simulation is close to $\omega_{\mathrm{hd}, i}$ in spite of the low concentration of impurities. The overall agreement between the simulation and the model is fairly good, and the deviations may be attributed to low-temperature effects and to shape deformations of the distributions not entering Eqs. (5).

Finally, it is worthwhile stressing that even though the effects on the dipolar oscillations due to the scattering with impurities are small, they can be greatly increased by tuning the experimental parameters. For instance, if we consider the parameters of the recent experiment by Takasu et al. 24] and take $100{ }^{174} \mathrm{Yb}$ atoms as impurities, the difference between the oscillation frequencies along the axial and radial directions as shown in Fig. 1 will be of about $75 \%$ in the limit of small $\lambda$. Therefore, we may expect that the effects here described will become easily observable in the near future when mixtures of ${ }^{40} \mathrm{~K}$ and ${ }^{174} \mathrm{Yb}$ will be experimentally realized.

\section{B. Monopolar and quadrupolar oscillations}

Dipolar modes are a simple example of collective modes that can be experimentally analyzed and whose features expose the collisional state of the gas. As illustrated by the classical model, these modes can be viewed mainly as coupled oscillations of the centers of mass of the two clouds in the absence of appreciable deformations of their shapes. Other collective modes can be explored by deforming the clouds in different ways. The lowestfrequency modes of this type are the surface monopolar $(l=0)$ and quadrupolar $(l=2)$ modes, and these can be excited by small deformations of suitable symmetry. While in an isotropic trap the monopolar mode and the $\ell_{z}=0$ quadrupolar mode can be independently excited, in an axially symmetric trap the angular momentum is not a good quantum number and the two oscillations with $\ell_{z}=0$ are coupled to each other. 

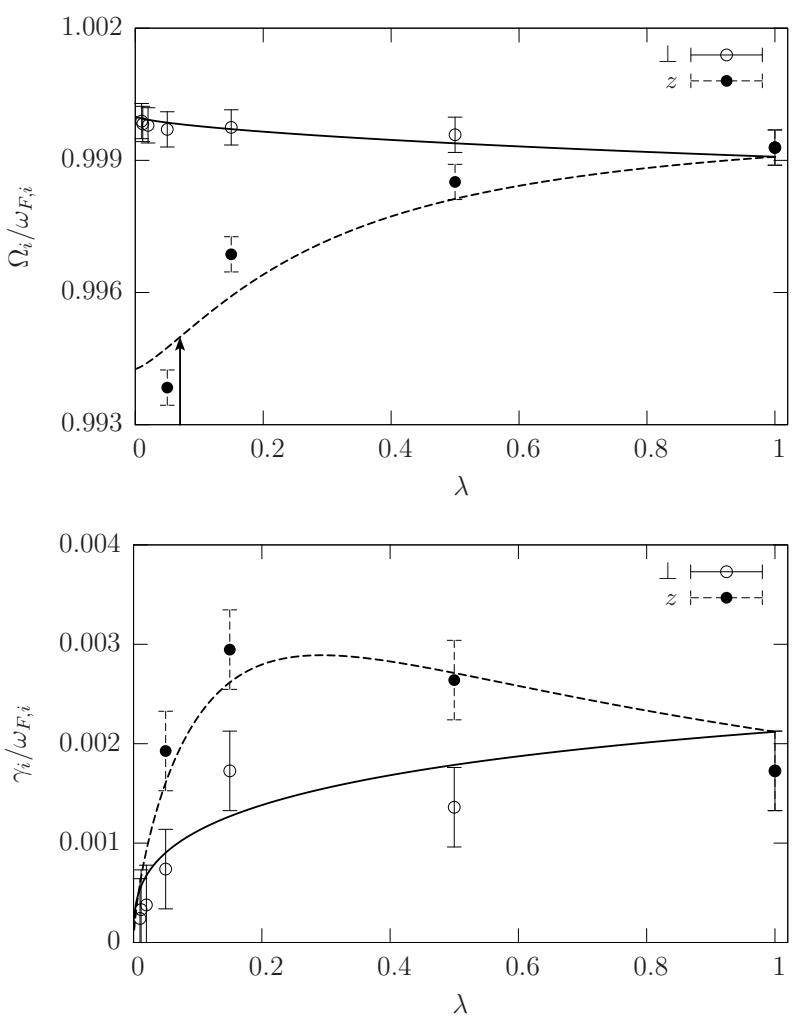

FIG. 1: Fermion dipolar frequencies $\Omega_{i}$ (top panel) and damping rates $\gamma_{i}$ (bottom panel) in units of $\omega_{F, i}$, as functions of the trap anisotropy $\lambda$. The results of the simulation (symbols with error bars) are compared with those obtained from Eqs. (5): open circles and solid lines denote radial oscillations, while full circles and dashed lines refer to motions along the $\hat{z}$ axis. The arrow in the top panel marks the locking point given in Eq. (9).

As the collisionality of the fermion gas is increased, the transition to the hydrodynamic regime in strongly elongated traps manifests itself through changes in the $\ell_{z}=0$ mode frequencies from $2 \omega_{F, z}$ to $\Omega_{\mathrm{hd}}=\sqrt{12 / 5} \omega_{F, z}$ and from $2 \omega_{F, \perp}$ to $\sqrt{10 / 3} \omega_{F, \perp}[25,[26,[27]$. A similar transition can be expected in the present system, where the impurities act to transfer momentum between noninteracting fermions. We have monitored several dynamical averages of the fermion cloud in the course of the simulation and in particular we have analyzed the time evolution of $\chi(t)=\left\langle 2 v_{F, z}^{2}-v_{F, \perp}^{2}\right\rangle$ after compression of the cloud density by about $5 \%$. This average measures the anisotropy of the velocity distribution and is the responsible for the coupling between monopole and quadrupole excitations 27]. At variance from the dipole modes, in the monopole and quadrupole modes the radial and axial motions are strongly coupled and this requires that we simultaneously follow the dynamics of the gas on two quite different time scales. In Fig. 2 we plot the value of the lower $\ell_{z}=0$ mode frequency obtained from the Fourier transform of $\chi(t)$. At very large anisotropy this peak frequency suddenly drops from the collisionless value $2 \omega_{F, z}$

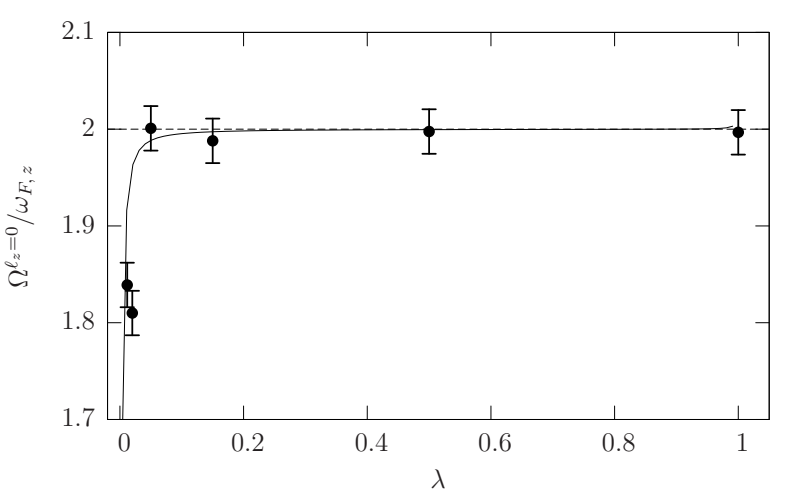

FIG. 2: Frequency $\Omega_{0}$ (in units of $\omega_{F, z}$ ) of the lower oscillation mode with $\ell_{z}=0$, as a function of $\lambda$ (symbols with error bars giving standard deviations). The solid line shows the lower $\ell_{z}=0$ frequency for a interacting fermion system with $\bar{\omega}_{F} \tau_{m q}=15$ (see text).

towards the hydrodynamic value $\sqrt{12 / 5} \omega_{F, z}$, whereas the higher peak frequency (not shown in Fig. 2) stops at its collisionless value $2 \omega_{F, \perp}$. Again collisionless and hydrodynamic behaviors coexist in a strongly elongated trap.

The behavior of monopolar and quadrupolar modes with finite relaxation time $\tau_{m q}$ in a gas of interacting fermions inside an anisotropic trap has been studied by Vichi 25], who derived an implicit polynomial equation predicting a very steep downturn of the lower mode frequency in systems with $\bar{\omega}_{F} \tau_{m q} \gg 1$. The solid line in Fig. 2 shows the result of fitting Vichi's model to our data with the choice $\bar{\omega}_{F} \tau_{m q}=15$. The sharp transition to the collisional regime is due to the fact that the impurities mediate the fermion-fermion scattering and lead to an effective relaxation time which is larger than that involved in the impurity-fermion scattering. Even though the effect is limited to large anisotropies, it could become observable at moderate anisotropies by increasing the numbers of particles or the strength of the bosonfermion repulsion.

\section{EXPANSION DYNAMICS}

Many experiments extract information on the properties of an ultracold trapped gas after it been allowed to undergo free expansion. This improves the spatial resolution of in-situ measurements and also gives access to the momentum distribution of the atoms. A fermion gas in the collisionless regime becomes spatially isotropic as it expands, regardless of the initial anisotropy of its density profile, whereas in the hydrodynamic regime the density profile inverts its aspect ratio during expansion. Furthermore, since the number of collisions diminishes as the gas expands, the hydrodynamic picture will become invalid when there are not enough collisions to sustain local equilibrium. 


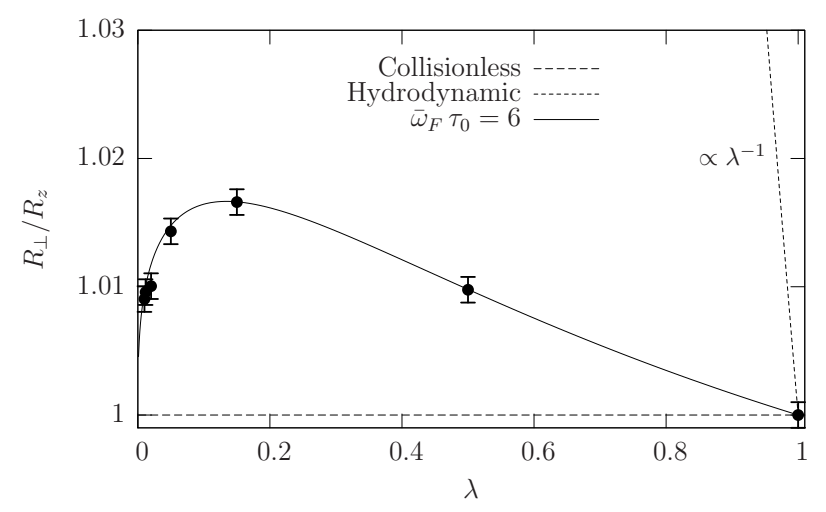

FIG. 3: Asymptotic aspect ratio $R_{\perp} / R_{z}$ of the expanded fermion cloud as a function of the trap anisotropy $\lambda$. The error bars are estimated from standard deviations in the fitting process. The solid line is the solution of Eq. (11) with $\tau_{0}=6 / \bar{\omega}_{F}$. The collisionless and hydrodynamic limits are shown by the dashed and short-dashed lines, respectively.

In the numerical simulation of the expansion, starting from the equilibrium density profiles of the trapped gaseous mixture we switch off the confinement and allow evolution according to the VLE. During the evolution we adaptively change the size of the computational domain in order to ensure that all particles are inside it. This permits us to study the free expansion of the cloud for long periods of time up to expansion ratios $b_{i}(t)=R_{i}(t) / R_{i}(0)$ of a few hundreds, with $R_{i}(t)=\left\langle x_{F, i}^{2}(t)\right\rangle^{1 / 2}$ being the width of the cloud in the $i$-direction. In Fig. 3 we show the asymptotic value $R_{\perp} / R_{z}$ of the aspect ratio of the fermion cloud as a function of the anisotropy $\lambda$. The aspect ratio in the case of isotropic or strongly anisotropic confinement is equal to unity as in the collisionless regime, while for intermediate values of $\lambda$ the scattering against the impurities makes $R_{\perp} / R_{z}$ deviate from unity. This behavior can be seen as a consequence of the competition between the time scales associated to axial and radial motions, as explained below.

We have evaluated the total numbers $\mathcal{Q}_{\perp}$ and $\mathcal{Q}_{z}$ of collisions occurring during a lapse of time from $t=0$ to $t_{\perp}=1 / \omega_{F, \perp}$ and to $t_{z}=1 / \omega_{F, z}$, respectively. Assuming that the collision rate $Q(t)$ scales in time with the volume occupied by the gas and that the expansion dynamics is close to that of a collisionless system, $\mathcal{Q}_{\perp}$ and $\mathcal{Q}_{z}$ can be written as

$$
\begin{aligned}
\mathcal{Q}_{\perp, z} & =\int_{0}^{t_{\perp}, t_{z}} d t Q(t) \\
& =Q(0) \int_{0}^{t_{\perp}, t_{z}} d t\left(1+\omega_{F, \perp}^{2} t^{2}\right)^{-1}\left(1+\omega_{F, z}^{2} t^{2}\right)^{-1 / 2} .
\end{aligned}
$$

In Eq. (11) we have taken $Q(t)=Q(0) /\left(b_{\perp}^{2} b_{z}\right)$ and set $b_{i}(t)=\left(1+\omega_{F, i}^{2} t^{2}\right)^{1 / 2}$ [28]. The integral in Eq. (11) is straightforward and yields the results shown in Fig. 4 The following points should be noted: (i) the characteristic time $t_{\perp}$ for the radial expansion and consequently $\mathcal{Q}_{\perp}$ decrease with increasing the anisotropy; and

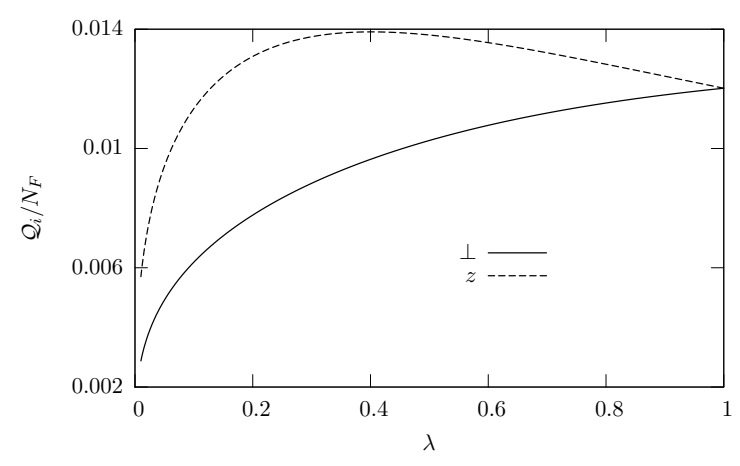

FIG. 4: Numbers $\mathcal{Q}_{\perp} / N_{F}$ (solid line) and $\mathcal{Q}_{z} / N_{F}$ (dashed line) of collisions per fermion, as functions of the anisotropy parameter $\lambda$.

(ii) the integrand of Eq. (11) for $\mathcal{Q}_{z}$ becomes negligible for $t_{\perp} \ll t \leq t_{z}$ as the fermion density drops due to rapid expansion in the radial direction, so that $\mathcal{Q}_{z}$ increases at first with decreasing $\lambda$, reaches its maximum at $\lambda \simeq 0.4$, and then rapidly drops as the density of the expanding cloud rapidly vanishes.

The non-monotonic behavior of $R_{\perp} / R_{z}$ as a function of $\lambda$ in Fig. 3 can be understood from the features of $\mathcal{Q}_{\perp}$ and $\mathcal{Q}_{z}$ in Fig. 4 . The number $\mathcal{Q}_{\perp}$ of collisions diminishes with decreasing $\lambda$ while $\mathcal{Q}_{z}$ increases, so that collisions play different roles in the axial and radial expansion. Both $\mathcal{Q}_{\perp}$ and $\mathcal{Q}_{z}$ are dropping at large anisotropies, so that collisionless behavior is emerging in the ballistic expansion from a strongly elongated trap.

The behavior of the expanding gas as a function of $\lambda$ can also be analyzed by means of scaling equations. In this approach [28] the expansion of a fermion gas with finite collisionality can be described by the equations

$$
\left\{\begin{array}{l}
\ddot{b}_{i}-\omega_{F, i}^{2} \frac{\theta_{i}}{b_{i}}=0 \\
\dot{\theta}_{i}+2 \frac{\dot{b}_{i}}{b_{i}} \theta_{i}^{2}=\frac{1}{\tau\left(\theta_{i}, b_{i}\right)}\left(\theta_{i}-\frac{1}{3} \sum_{j} \theta_{j}\right)
\end{array}\right.
$$

where $\theta_{i}(t)$ are effective temperatures along the two spatial directions, $\tau\left(\theta_{i}, b_{i}\right)=\tau_{0}\left(\prod_{j} b_{j}\right)\left(\frac{1}{3} \sum_{k} \theta_{k}\right)^{-1 / 2}$ and $\tau_{0}$ is the collision time at $t=0$. The collisionless and hydrodynamic limits correspond to taking $\tau=\infty$ and $\tau=0$ in Eqs. (11), respectively.

We have taken the collision time $\tau_{0}$ in Eqs. (11) as a fitting parameter to represent the indirect fermion-fermion scattering induced by the impurities. The solution of Eqs. (11) with the choice $\tau_{0}=6 / \bar{\omega}_{F}$ reproduces the nonmonotonic behavior of the aspect ratio as shown in Fig. 3 Finally, we have checked that the qualitative behavior shown in Fig. 4 for $\mathcal{Q}_{\perp}$ and $\mathcal{Q}_{z}$ does not depend on the assumption of a collisionless evolution of the gas and persists on describing its expansion with any value of $\tau_{0}$. The magnitude of the deviation from unity of the aspect ratio of the expanding fermions depends on the value of 
$\tau_{0}$ and can be increased by changing the fermion-impurity interaction via Feshbach resonances 29] or by performing an out-of-equilibrium experiment as described in Ref. [20].

\section{SUMMARY AND CONCLUDING REMARKS}

We have studied the low-lying surface modes and the ballistic expansion of a spin-polarized Fermi gas interacting with thermal impurities as functions of the anisotropy of its confinement in a cigar-shaped harmonic trap. We have solved for this purpose the Vlasov-Landau equations for the dynamics of the mixture and compared the results with simple scaling equations containing collisiontime fitting parameters. The results show that for large anisotropies a collisionless behavior in the radial dipolar oscillations and a hydrodynamic behavior in the axial ones are simultaneously established. For monopolar and quadrupolar excitations we have observed a collisionless spectrum irrespectively of the strength of the anisotropy parameter, except for extremely large anisotropies where the frequency of the lower $\ell_{z}=0$ mode decreases towards the hydrodynamic value.

On the other hand, during ballistic expansion the two different time scales for radial and axial motions enter into competition. The result is that the expansion of a strongly anisotropic cloud is essentially collisionless due to the rapid drop of the particle density, whereas at intermediate values of the anisotropy the aspect ratio is sensitive to the collisions.

The analysis presented here has only concerned slightly doped fermions in a collisionless-to-intermediate scattering regime as, e.g., that attained in the experiments at LENS. The effects of the trap anisotropy could be further enhanced exploiting the rich variety of experimental set-ups and trapped isotopes available. It would be also interesting to extend our work to strong-interaction situations among fermions and impurities or among fermions in two-component Fermi gases approaching the unitary limit.

\section{Acknowledgments}

This work has been partially supported by an Advanced Research Initiative of Scuola Normale Superiore di Pisa and by the Istituto Nazionale di Fisica della Materia within the Initiative "Calcolo Parallelo".
[1] A. Minguzzi, S. Succi, F. Toschi, M. P. Tosi, and P. Vignolo, Phys. Rep. 395, 223 (2004).

[2] M. H. Anderson, J. R. Ensher, M. R. Matthews, C. E. Wieman, and E. A. Cornell, Science 269, 198 (1995).

[3] C. C. Bradley, C. A. Sackett, J. J. Tollett, and R. G. Hulet, Phys. Rev. Lett. 75, 1687 (1995).

[4] K. B. Davis, M. O. Mewes, M. R. Andrews, N. J. van Druten, D. S. Durfee, D. M. Kurn, and W. Ketterle, Phys. Rev. Lett. 75, 3969 (1995).

[5] B. DeMarco and D. S. Jin, Science 285, 1703 (1999).

[6] A. G. Truscott, K. E. Strecker, W. I. McAlexander, G. B. Partridge, and R. G. Hulet, Science 291, 2570 (2001).

[7] F. Schreck, L. Khaykovich, K. L. Corwin, G. Ferrari, T. Bourdel, J. Cubizolles, and C. Salomon, Phys. Rev. Lett. 87, 080403 (2001).

[8] J. Goldwin, S. B. Papp, B. DeMarco, and D. S. Jin, Phys. Rev. A 65, 021402 (2002).

[9] K. M. O'Hara, S. L. Hemmer, M. E. Gehm, S. R. Granade, and J. E. Thomas, Science 298, 2179 (2002).

[10] M. Greiner, C. A. Regal, and D. S. Jin, Nature 426, 537 (2003).

[11] C. Chin, M. Bartenstein, A. Altmeyer, S. Riedl, S. Jochim, J. Hecker Denschlag, and R. Grimm, Science 305, 1128 (2004).

[12] R. Graham and D. Walls, Phys. Rev. A 57, 484 (1998).

[13] P. Capuzzi, A. Minguzzi, and M. P. Tosi, Phys. Rev. A 67, 053605 (2003).

[14] P. Capuzzi, A. Minguzzi, and M. P. Tosi, Phys. Rev. A 68, 033605 (2003).

[15] J. Kinast, S. L. Hemmer, M. E. Gehm, A. Turlapov, and J. E. Thomas, Phys. Rev. Lett. 92, 150402 (2004).
[16] M. Bartenstein, A. Altmeyer, S. Riedl, S. Jochim, C. Chin, J. Hecker Denschlag, and R. Grimm, Phys. Rev. Lett. 92, 203201 (2004).

[17] J. Kinast, A. Turlapov, and J. E. Thomas (2004), condmat/0408634.

[18] S. D. Gensemer and D. S. Jin, Phys. Rev. Lett. 87, 173201 (2001).

[19] F. Toschi, P. Vignolo, S. Succi, and M. P. Tosi, Phys. Rev. A 67, 041605 (2003).

[20] P. Capuzzi, P. Vignolo, F. Toschi, S. Succi, and M. P. Tosi, Phys. Rev. A 70, 043623 (2004).

[21] S. Succi, F. Toschi, P. Capuzzi, P. Vignolo, and M. P. Tosi, Phil. Trans. R. Soc. London A 362, 1605 (2004).

[22] F. Ferlaino, R. J. Brecha, P. Hannaford, F. Riboli, G. Roati, G. Modugno, and M. Inguscio, J. Opt. B 5, S3 (2003).

[23] L. Vichi and S. Stringari, Phys. Rev. A 60, 4734 (1999).

[24] Y. Takasu, K. Maki, K. Komori, T. Takano, K. Honda, M. Kumakura, T. Yabuzaki, and Y. Takahashi, Phys. Rev. Lett. 91, 040404 (2003).

[25] L. Vichi, J. Low. Temp. Phys. 121, 177 (2000).

[26] A. Griffin, W.-C. Wu, and S. Stringari, Phys. Rev. Lett. 78, 1838 (1997).

[27] D. Guéry-Odelin, F. Zambelli, J. Dalibard, and S. Stringari, Phys. Rev. A 60, 4851 (1999).

[28] P. Pedri, D. Guéry-Odelin, and S. Stringari, Phys. Rev. A 68, 043608 (2003).

[29] S. Inouye, J. Goldwin, M. L. Olsen, J. L. Bohn, and D. S. Jin, Phys. Rev. Lett. 93, 183201 (2004). 